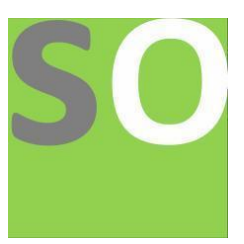

Article title: A Physical Theory of Information vs A Mathematical Theory of Communication Authors: Manouchehr Amiri[1]

Affiliations: Tehran Azad University[1]

Orcid ids: 0000-0002-0884-8853[1]

Contact e-mail: manoamiri@gmail.com

License information: This work has been published open access under Creative Commons Attribution License http://creativecommons.org/licenses/by/4.0/, which permits unrestricted use, distribution, and reproduction in any medium, provided the original work is properly cited. Conditions, terms of use and publishing policy can be found at https://www.scienceopen.com/.

Preprint statement: This article is a preprint and has not been peer-reviewed, under consideration and submitted to ScienceOpen Preprints for open peer review.

DOI: 10.14293/S2199-1006.1.SOR-.PPVROKO.v1

Preprint first posted online: 17 October 2020

Keywords: A Physical theory of information, Binary data matrix model, Shannon information theory, Bit information principle 


\title{
A Physical Theory of Information
}

\author{
Manouchehr Amiri ${ }^{1}$, Tehran Azad University
}

\begin{abstract}
In this article, I develop a general notion of physical bit information which is compatible with the basics of quantum mechanics and incorporates the Shannon entropy as a special case. This notion of physical information leads to Binary data matrix model (BDM) which predicts the basic results of quantum mechanics, general relativity, and black hole thermodynamics. the compatibility of the model with holographic, information conservation and Landauer's principles is investigated. After deriving the "Bit Information principle" as a consequence of BDM, the fundamental equations of Planck, De Broglie, Beckenstein and mass-energy equivalence are derived.
\end{abstract}

Keywords: Physical theory of information, Binary data matrix model, Shannon information theory, Bit information principle

\section{Introduction}

Information in a broad sense implies a collection of data of unmeasurable concepts or measurable quantities. The usual notion of measurable information in physics invokes the subject of Shannon entropy and information. Claude Shannon in his seminal paper [1] developed a mathematical theory of signal transmission [2]. He denied the semantic aspects of communication and related information theory. According to his theory, the information refers to the opportunity to reduce uncertainty and equals the entropy of the communicated message. He had got the idea of entropy from the second law of thermodynamics [2,3] and concluded that the information of a message could be measured by its predictability, the less predictability the more information it carries $[2,3]$. Shannon was quite clear that his definition was not the unique definition of information but merely one definition of information suited for his engineering requirements [3]. In this notion of information, the source, channel and receiver of data are crucial components of a communication engineering. Shannon entropy (information) is just concerned with the statistical properties of a given system, independent of the meaning and semantic content of system states [5]. As he emphasized in his seminal article, the meaning of communication and related information content are irrelevant to engineering problem [1]. Subsequently, there have been emerged some critiques around the Shannon notion of physical and biological information [3]. The notion of information independent of its meaning is the subject of main criticism announced by MacKay and others $[3,4]$. Subsequently there have been attempts to add a semantic dimension to a formal theory of information, particularly to Shannon's theory $[5,6,7]$. Shannon theory is not concerned with individual message but rather the averages of source messages [8]. Although the physical information basically is related to physical measurable quantities, the current notion of physical information remained as the same definition introduced by Shannon and seems to be insufficient for physical systems. This has been reminded in recent works of Bruckner and Zeilinger [9]. Their main reason for this claim is the measurement problem in quantum mechanics. in other words, there is no definite real value for observables before measurement in quantum physics sense and

\footnotetext{
${ }^{1}$ manoamiri@gmail.com
} 
there is no reality independent of observer or measurement [9]. In quantum information theory Von Neumann entropy is a candidate for replacing Shannon entropy to measure quantum information [9]. However recent trends toward deterministic quantum mechanics and its local versions $[10,11,12]$ motivates one to investigate the existence of a fundamental version of information which carries the physical meaning of the system and is compatible with quantum mechanics. the main purpose of this article focused on introducing a new version of physical information and its broad consequences. In this version of information theory that I call it "Physical Theory of Information" a bit of information reflects a real physical quantity in phase space, and it does not concern with the observer dependent reality of physical parameters.

\section{Binary Matrix Model}

Does information do anything with physics? The response of J. A. Wheeler was "It from bit" which means that entire universe constituted from "bits". Generally, information is defined as "an answers to specific question" [3]. Therefor if we restrict answers to "Yes" or "No" the information could be represented by a set of 0 and 1 or a binary array. The formalism of information can be generalized by starting from this more detailed notion of information i.e. the arrays of binary data 0 and 1 for each physical parameter of particles in a system. Any variable (physical parameter) $x_{v}$ when attributed to a subject or object (particles), carries the information that reflects the "quantity" of that variables. $v$ refers to the specific parameter and varies between 1 and $d$ ( degree of freedom or). If we divide the range of this variable into a large number of infinitesimal intervals, then the value of variable is represented by a binary column matrix with 1 entry at the interval where the value of variable $x_{v}$ restricted, as depicted in fig. 1

$$
x_{v \downarrow} \uparrow\left[\begin{array}{c}
0 \\
0 \\
\vdots \\
1 \\
\vdots \\
0
\end{array}\right] \rightarrow x_{v 0}
$$

Fig.1 The column matrix's entries return 0 except for the entry which corresponds to the value $x_{v 0}$ of parameter $x_{v}$ and represented by 1 .

The unique entry 1 corresponds the specific interval that represents the quantity $x_{v 0}$ of variable $x_{v}$.

$$
x_{v 0}-\delta x_{v}<x_{v}<x_{v 0}+\delta x_{v}
$$

Other values can be presented by displacing the 1 entry along the range of variable $x_{v}$ of column matrix. For a set of different variables there are similar column matrices. By consideration of a system of $\mathrm{N}$ identical particles ( $\mathrm{N}$ is a large number), for each particle there is a binary column matrix that specifies the $x_{v}$ parameter of that particle. Assembling all these column matrices results in a binary data matrix $D_{v}$ (Fig 2). As an example, the column matrix specified by red box, determines the value of $x_{v}$ for second particle (particle assigned by number2). The rows of $D_{v}$ are binary arrays $e^{* v}\left(x_{v 0}\right)$ which are defined at any point $x_{v 0}$ and returns the information of particles at that point, depicted by blue box in Fig.2:

$$
e^{* v}\left(x_{v 0}\right)=(0110 \ldots 10)
$$




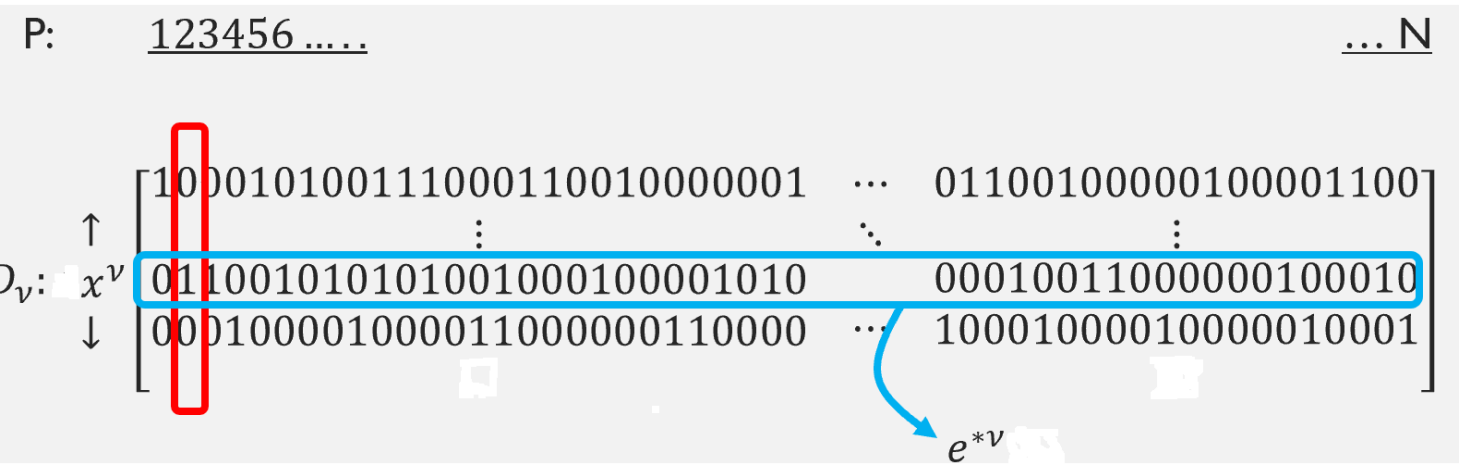

Fig2. The structure of matrix $D_{v}$. The red box contains a unique " 1 " at the specific value $x_{v 0}$ which is the parameter value of particle with label 2 . The blue box represents the particles whose parametric values is $x_{v 0}$. Any " 1 " entry in this array corresponds the particle with physical value $x_{v 0}$.

Any particle with the parameter value $x_{v 0}$ represented by entry 1 in this array. The sum of all 1 entries in $e^{* v}\left(x_{v 0}\right)$ gives the total number of particles whose parameter's value is $x_{v 0}$ simultaneously. In phase space, there are $d$ different phase space parameters i.e. degree of freedom (including spatial, linear, and angular momentum), which lead to different $D_{v}$. For a comprehensive binary information data of system, we embed all $D_{v}$ in a columnar matrix $D$ :

$$
D=\left[\begin{array}{c}
D_{1} \\
D_{2} \\
\vdots \\
D_{d}
\end{array}\right]
$$

Where $d$ is the degree of freedom. For any point $\left(x_{10}, x_{20}, \ldots x_{d 0}\right)$ on phase space, there correspond a set of $e^{* v}\left(x_{v 0}\right)$ that could be gathered in a matrix $P$ :

$$
P=\left[\begin{array}{c}
{\left[e^{* 1}\right]} \\
{\left[e^{* 2}\right]} \\
\vdots \\
{\left[e^{* d}\right]}
\end{array}\right]
$$

Where $\left[e^{* v}\right]$ are row binary matrices defined at the point $\left(x_{10}, x_{20}, \ldots x_{d 0}\right)$.

Theorem 1: the entries of matrix $G=\left\|g^{v \mu}\right\|=P P^{T}$ give the number of particles with the same values of $x_{i 0}$ and $x_{j 0}$ and $\frac{1}{N} f_{v \mu}$ represents the joint probabilities.

Proof: with the above definitions, it can be easily proved that the inner products

$$
g^{v \mu}=\left[e^{* v}\right]\left[e^{* \mu}\right]^{T}
$$

are the number of particles with the values $x_{v 0}$ and $x_{\mu 0}$ simultaneously. Obviously $G$ is symmetric. Dividing $g_{v \mu}$ by total particles number obtains the joint probabilities $f_{v \mu}$ :

$$
f^{v \mu}=\frac{1}{N} g^{v \mu}
$$

Matrix $D$ contains all physical information of system constituents at any time. for a dynamical system $D$ evolves over time while the total number of " 1 " bit preserved. This number that reflects 
the information measure of the system is the product of total number of particles and degree of freedom: $\quad \mathbb{I}=\mathrm{Nd}$

Which is a constant. This formalism of physical bit information is called BDM (Binary Data Matrix Model) and ensues three immediate consequences:

a) The invariance of $\mathbb{I}$ is compatible with information conservation principle.

b) If the system's temperature denoted by $T$, then the average energy per particle due to equipartition principle reads as:

$$
\epsilon=\frac{d}{2} k T
$$

Since any particle contain $d$ bits, therefor the energy per bit could be obtained by dividing $\epsilon$

by $d$ : $\quad \varepsilon \cong \frac{1}{2} k T$

This is compatible with Landauer's principle i.e. $\varepsilon=k T \log 2$

c) All information of system represented by a 2-dimensional binary matrix $D$. This is compatible with Holographic principle.

These are physical principles that correlates the pure physical concepts to bit information and could not be justified in the context of Shannon information theory.

\section{Consequences of BDM}

If we Consider $\left[e^{* v}\right]$ as the dual base vectors in the parameter space (phase space), the inner product (4) gives the dual metric tensor of this space. Author in his previous work [13] proved that the evolution of this metric tensor over time, obeys the quantum Liouville equation and leads to the equation (by assumption natural unit i.e. $\hbar=1$ ) [13]:

$$
\langle E\rangle=\frac{1}{2} \frac{\partial}{\partial \tau} \log g
$$

$g$ stands for determinant of $\left\|g^{v \mu}\right\|$ and $\langle E\rangle$ for energy per system constituents i.e. particles. On the other hand, for spatial displacement, we obtain the corresponding equation [13]:

$$
p_{i}=-\frac{1}{2} \frac{\partial}{\partial x_{i}} \log g
$$

The term $-\frac{1}{2} \log g$ shows the Hamilton's principal function $F$ in classical mechanics [15]:

$$
E=H=-\frac{\partial F}{\partial \tau} \quad ; \quad p_{i}=\frac{\partial F}{\partial x_{i}}
$$

More extension of BDM results in a general form of Einstein Field Equation and Schrodinger wave equation [13]. This results are not considered in present article.

\subsection{Entropy and Information}

In classical statistics, for jointly normal random variables $\boldsymbol{x}_{1}, \boldsymbol{x}_{2}, \ldots \boldsymbol{x}_{n}$, the entropy is

calculated as [14]: $\quad \mathcal{H}=\frac{1}{2} \log \Delta+k$

Where $\Delta$ is the determinant of inverse of covariance matrix $\left(\sigma^{i j}\right)$ i.e. $\Delta=\operatorname{det} \sigma_{i j}$ and $k$ is constant $k=\log 2 \pi e$. We show at the limit $\sigma \rightarrow 0$ ( $\sigma$ is variance of random variables) as can be 
realized in the case of black holes, we can replace the Fisher metric [14] with metric of BDM model.

Theorem 2: At the limit $\sigma \rightarrow 0$ as can be realized in the case of black holes [16], inverse of Fisher metric determinant and determinant of BDM metric are identical.

Proof: In the case of black holes where all the physical variables of its constituent confined to constant infinitesimal intervals $\delta x^{i}$, around the singularity point, if we fix the center of mass of black hole on the origin of spatial coordinates, the expected values of position and momentum of constituents are near zero corresponding BDM metric will be concentrated over these intervals with negligible values out of $\delta x^{i}$ and mean values near to zero. Thus, the correlation (covariance) matrix element $\Re_{i j}$ while the mean of all random variables vanishes $\bar{x}=0$ reads as:

$$
\Re_{i j}=\sigma_{i j}=\left\langle g_{i j} \delta x_{i} \delta x_{j}\right\rangle=g_{i j} \delta x_{i} \delta x_{j}
$$

Determinant of $\sigma_{i j}$ matrix (denoted by $\Delta$ ) could be calculated as:

$$
\Delta=\sum_{i j k \ldots} \varepsilon_{i j k \ldots} \sigma_{i 1} \sigma_{j 2} \sigma_{k 3} \ldots
$$

Substitution of $\sigma_{i j}$ with $g_{i j} \delta x_{i} \delta x_{j}$ gives rise to:

$$
\Delta=g \prod_{i}\left(\delta x_{i}\right)^{2}
$$

Logarithm of both sides results in:

$$
\log \Delta=\log g+2 \sum_{i} \log \delta x_{i}=\log g+C
$$

On the other hand, definition of Fisher information metric $g_{i j}$ results in the equivalence of inverse of covariance matrix $\sigma^{i j}$ and fisher metric tensor:

$$
g_{i j}=\left(\sigma_{i j}\right)^{-1}=\sigma^{i j}
$$

If determinant of $g_{i j}$ denoted by $g$, then by equation (15) and (16) we have:

$$
g=\Delta^{-1} \quad ; \quad \log g^{-1}=\log g+C^{\prime}
$$

and theorem is proved.

Therefor the equation (11) for entropy can be replaced by

$$
\mathcal{H}=\frac{1}{2} \log g^{-1}+C=\frac{1}{2} \log g+C^{\prime \prime}
$$

The equivalence of entropy $\mathcal{H}$ and $\frac{1}{2} \log g$ results in "Bit Information Principle".

\subsection{Bit Information Principle}

The equation (18) implies that the entropy $\mathcal{H}$ is equivalent to $\frac{1}{2} \log g$. By definition, the entropy is equivalent to the measure of information, then we conclude an important result which I call it 
"Bit Information principle": $\frac{1}{2} \log \Delta$ is a measure of information at the limit $\sigma \rightarrow 0$ and its spatial and temporal densities give the expected energy and momentum per particle.

to conclude this principle, first consider the equation (18). It implies the entropic nature of $\frac{1}{2} \log g$ at the limit $\sigma \rightarrow 0$. Assuming equivalence of entropy and information, if we denote information by $\mathbb{I}$, we get: $\quad \mathbb{I}=\frac{1}{2} \log g$

Respect to equations (8) and (9) we infer that the time and spatial derivatives of $\frac{1}{2} \log g$ obtains $\langle E\rangle$ and $p_{i}$ as the expected energy and momentum of system constituents. After returning to MKS units by multiplying the equations by $\hbar$ (Planck constant) we get:

$$
\langle E\rangle=\hbar \frac{\partial}{\partial \tau} \mathbb{I} ; p_{i}=-\hbar \frac{\partial}{\partial x_{i}} \mathbb{I}
$$

In BDM model, the information I, is the number of bits. Therefor the equations (20) reveal the relation between the density of bits over spatial and temporal intervals and momentum and energy per constituent of system. In a brief notation:

$$
\text { energy } \sim \frac{\text { bits }}{\text { second }}, \text { momentum } \sim \frac{\text { bits }}{\text { unit length }}, \text { angular momentum } \sim \frac{\text { bits }}{\text { unit angle }}
$$

Any system with negligible variance of its constituents' parameters, exhibits a set of identical bits. For example, a monochromatic electromagnetic or acoustic wave, possess a set of identical full wavelengths as bits.

\subsection{Outcomes of Bit Information Principle}

This principle justifies the Planck and De Broglie equation. When all bits are identical, it means that $\sigma \rightarrow 0$ and the required condition for Bit Information Principle is met. The situation of identical bits can be realized in monochromatic light (electromagnetic wave) beam because every full wavelength (pulse) should be considered as a bit of information. For temporal (time) density of these pulse, the number of bits (full wavelength) per unit time is:

$$
f=\frac{1}{T}
$$

Where $T$ is the period of monochromatic wave. Then due to (20) we have:

$$
\langle E\rangle=\hbar \frac{1}{T}=\hbar f
$$

This is the Planck formula for energy of light wave constituents which is called photons. For spatial density of these pulse, the number of full wavelength (bit) per unit length is:

$$
n=\frac{1}{\lambda}
$$

Then respect to (20) and ignoring the sign, we obtain:

$$
p=\frac{\hbar}{\lambda}
$$


This is the De Broglie relation for wavelength and momentum of a particle. The same relations are also valid for phonons as the quanta of mechanical waves. For a black hole the conditions for bit information principle is also met because all the mass and its constituents are confined in a infinitesimal interval of space and momentum and consequently their variances tends to zero, $\sigma \rightarrow 0$. What amount of mass is the "bit" of a black hole? Theoretically the mass of smallest possible black hole respect to limitations imposed by Schwarzschild radius, is the Planck mass:

$$
m_{p}=\sqrt{\frac{\hbar c}{G}}
$$

Then for a macroscopic body like black hole, the number of containing bits should be derived by dividing its mass to Planck mass:

$$
n=\frac{m}{m_{p}}
$$

Due to bit information principle, the energy is equivalent to bit density over time. The universal time unit (the least time interval) is the Planck time:

$$
t_{p}=\sqrt{\frac{G \hbar}{c^{5}}}
$$

Therefor the expected energy will be obtained by density of $n$ bits in (25) over the Planck time (27) multiplied by $\hbar$ :

$$
E=\hbar \frac{n}{t_{p}}=\hbar \frac{m}{m_{p}} \frac{1}{t_{p}}=\hbar m \sqrt{\frac{G}{\hbar c}} \sqrt{\frac{c^{5}}{G \hbar}}=m c^{2}
$$

This is the Einstein's mass-energy relation.

The bit information principle is also valid for linear and angular pseudo-momentum in crystals. The ordered arrangement of atoms, ions and molecules in a crystalline material provides the required conditions for the principle. Ant atomic planes in a crystal contains atoms which are confines in a small interval of space and momentum. Hence these atoms can be considered as bits over the interval of the interplanar surfaces of atoms. For bit density along the spatial coordinates in crystal lattices, the total density of a plane of atoms along the axis perpendicular to that plane, is proportional to $\frac{1}{d}$ where $d$ is the distance between atoms planes in crystal. The magnitude of corresponding reciprocal base lattice is also $\frac{1}{d}$ :

$$
|G|=\frac{1}{d}
$$

The vector with this magnitude perpendicular to the atoms plane is called crystal momentum or pseudo-momentum. This momentum appears just in the interactions of atoms lattice with an incident photon or particle waves. Respect to the bit density principle from previous section, this pseudo-momentum is equal to density of bits (atoms) over the interval $d$ by the equation:

$$
\mathcal{P}=\frac{\text { bits }}{\text { unit length }}=\frac{n}{d}
$$

The related momentum is the product of density and $\hbar$ i.e. 


$$
\mathcal{P}=\hbar \frac{n}{d}
$$

Then the pseudo-momentum per atom reads as:

$$
\mathcal{P}=\hbar \frac{1}{d}=\hbar G
$$

This is the main relation for crystal momentum which derived by the bit information principles.

Curiously, the similar relation should govern the angular momentum. The suggested relation is as

follows: $\quad L_{\theta}=\frac{\hbar}{\theta}$

Where $\theta$ is the angular period of bits over the whole $2 \pi$ as unit angle. For crystals with $N$-fold rotational symmetries it has been verified that the difference of pseudo angular momentum of incident and diffracted photons on a crystal with $\mathrm{N}$-fold rotational symmetries obey the relation [17]:

$$
\Delta m \hbar=\sigma \hbar+N P \hbar
$$

And for Rayleigh scattering with $\sigma_{i}$ and $\sigma_{s}$ as incident and scattered helicity of photons we have [17]:

$$
\sigma_{i}-\sigma_{s}=N P
$$

Where $P$ denotes an integer and $N$ determines the particles or bits density per unit angle $2 \pi$ (recall the definition of $N$-fold rotational symmetries). Thus, the equation (33) in BDM context is compatible with pseudo-angular momentum relation in (34) and (35).

Beckenstein Bound is another consequence of bit information principle. Due to this bound, the maximum information confined in a region with radius $R$ and energy $E$ due to Beckenstein is:

$$
I \leq \frac{2 \pi R E}{\hbar c \ln 2}
$$

In the language of bit information principle, the time span for calculation of bit information density, is the interval by which the light travels from center of black hole (singularity point) to horizon at the radius $R: \quad \Delta t=\frac{R}{c}$

Hence, the bit density over this interval is proportional to energy $E$ up to a coefficient $\hbar$ :

$$
E \simeq \hbar \frac{I}{\Delta t}=\hbar \frac{I c}{R} \Rightarrow I \simeq \frac{R E}{\hbar c}
$$

Which is compatible with (36).

\section{Conclusion}

By introducing a physical based theory of information, this article proves the authenticity and benefits of a new approach to the information concept in physics. This notion of information reduces to Shannon information as a special case. The BDM theory approach to information, not only endows a meaning to information, but also is compatible with the well-known principles such as Landauer's principle, conservation of information and Holographic principles. After deriving the 
crucial "bit information principle", The theory predicts the diverse principles such as, De Broglie, Planck and Beckenstein equations and mass-energy equivalence.

\section{References}

1) Shannon, Claude Elwood. "A mathematical theory of communication." ACM SIGMOBILE mobile computing and communications review 5.1 (2001): 3-55.

2) Griffin, Emory A. A first look at communication theory. McGraw-Hill, 2003.

3) Logan, Robert K. "What is information?: Why is it relativistic and what is its relationship to materiality, meaning and organization." Information 3.1 (2012): 68-91.

4) MacKay, D. M. "Proceedings of the information theory symposium." (1950).

5) Lombardi, Olimpia, Federico Holik, and Leonardo Vanni. "What is Shannon?" Synthese 193.7 (2016): 1983-2012.

6) Barwise, Jon, and Jerry Seligman. Information flow: the logic of distributed systems. Cambridge University Press, 1997.

7) Dretske, Fred. "Knowledge and the Flow of Information." (1981).

8) Timpson, Christopher Gordon. "Quantum information theory and the foundations of quantum mechanics." arXiv preprint quant-ph/0412063 (2004).

9) Brukner, Časlav, and Anton Zeilinger. "Conceptual inadequacy of the Shannon information in quantum measurements." Physical Review A 63.2 (2001): 022113.

10) Lindgren, Jussi, and Jukka Liukkonen. "The Heisenberg Uncertainty Principle as an Endogenous Equilibrium Property of Stochastic Optimal Control Systems in Quantum Mechanics." Symmetry 12.9 (2020): 1533.

11) Haag, Rudolf. Local quantum physics: Fields, particles, algebras. Springer Science \& Business Media, 2012.

12) Hooft, Gerard T. "Deterministic quantum mechanics: the mathematical equations." arXiv preprint arXiv:2005.06374 (2020).

13) Amiri, Manouchehr. "On Equivalence of Quantum Liouville Equation and Metric Compatibility Condition, a Ricci Flow Approach." (2016).

14) Papoulis, Athanasios, and S. Unnikrishna Pillai. Probability, random variables, and stochastic processes. Tata McGraw-Hill Education, 2002.

15) H. Goldstein, Classical mechanics: Pearson Education India, 1965

16) C.Castro, (2008). The Euclidean gravitational action as black hole entropy, singularities, and spacetime voids. Journal of Mathematical Physics, 49(4), 042501.

17) Tatsumi, Yuki, Tomoaki Kaneko, and Riichiro Saito. "Conservation law of angular momentum in helicity-dependent Raman and Rayleigh scattering." Physical Review B 97.19 (2018): 195444. 